\title{
Strategies for Early Diagnosis and Treatment of Coronary Heart Disease
}

\author{
Huizheng Zhu ${ }^{1}$ \\ ${ }^{1}$ Guangzhou University of Chinese Medicine, 510403
}

KEYWORDS: Early Diagnosis and Treatment; Coronary Heart Disease; Treatment Strategies

\begin{abstract}
Mainly refers to coronary heart disease coronary blood vessels atherosclerotic lesions, which lead to vascular stenosis or blocked, thereby causing myocardial ischemia and hypoxia or necrosis, which eventually led to a heart attack. According to relevant data show that the incidence of coronary heart disease is showing an increasing trend, affecting the quality of life of people and endanger their health. This study will be analyzed for the early diagnosis of coronary heart disease.
\end{abstract}

\section{Introduction}

Coronary heart disease, also known as ischemic heart disease, is a common heart disease, generally divided into five types, namely, angina, myocardial infarction, sudden death, ischemic heart disease, and silent myocardial ischemia. The clinical manifestations of coronary heart disease is mainly in the middle of the sternum or upper end episodes of chest pain, sometimes spread to the heart of the former district. Chest pain in patients with coronary heart disease, there will be oppression, nausea or tightening pain, burning sensation sometimes appear, patients have serious cause fear of dying, resulting in patients with shock, arrhythmia or heart failure, even death

Current methods have some value in the early diagnosis of coronary artery disease is the treadmill exercise test ECG, treadmill exercise test ECG stress test belongs, diagnosis of coronary heart disease is the most common way to assist, but this method is easy to check false negative or false positive. The 12-lead ECG synchronization method, according to the survey research shows that the current domestic synchronous 12-lead ECG, its scope has some limitations, only a part of the hospital use 12-lead ECG synchronization of their coronary heart disease early diagnosis. So normal ECG and normal on the relevant diagnostic criteria for various diseases, twelve lead ECG instrument still using traditional data. With the ever-changing social environment and people's living habits, 12-lead ECG synchronization using the old standard of its diagnosis in patients with coronary heart disease, the results prone to misdiagnosis, subject to certain accuracy questioned. In addition, early coronary angiography is the gold standard for diagnosis of coronary heart disease, coronary angiography for coronary artery can walk the line, as well as the number of deformity evaluation of coronary artery lesions, the severity and scope of the evaluation, while the coronal coronary angiography also functional changes and cardiac function evaluation, is a more effective method of diagnosing coronary heart disease, but its operation is more complex procedures and costs involved more.

\section{Evaluation of Coronary Heart Disease Diagnosis Method}

At present, the commonly used diagnostic methods include coronary heart disease symptoms, resting ECG, exercise ECG, stress echocardiography, myocardial perfusion imaging isotope load, coronary CT angiography, coronary angiography, etc., which are still being coronary angiography recognized as the gold standard for diagnosis of coronary heart disease. If these diagnostic methods 
are caused by excessive use of medical waste of resources, it is too small and cannot apply early and accurate diagnosis, how to rationalize and optimize application is difficult, it is necessary to make a systematic evaluation of their diagnostic value. Whether myocardial ischemia or myocardial infarction, the onset of clinical manifestations characteristic with characteristic ECG changes, coronary heart disease is the cornerstone of an important basis for the diagnosis and clinical symptoms of the onset of chest pain or pressure that is the location, nature, duration, and predisposing factors mitigation methods characteristic manifestations and ECG characteristic changes, especially in the early diagnosis of ACS is more important. After the onset of chest pain ECG ST-segment changes and if chest pain ST reply, it supports the diagnosis of myocardial ischemia angina; if typical dynamic evolution of myocardial infarction, the myocardial infarction can be established. But resting ECG diagnosis of chest pain in the non-coronary heart disease have limitations, cannot be normal, except when there is no attack of angina pectoris electrocardiogram, nonspecific ST-T changes without continuing evolution rather than at the onset of myocardial ischemia, not simply on clinical with resting ECG conclusion easily diagnosed myocardial ischemia.

Cardiac stress test. Cardiac stress test is a induced myocardial ischemia in patients with exercise or pharmacological stress state, while electrocardiogram, echocardiogram or radionuclide myocardial perfusion imaging methods such as observation and recording a series of checks. Load test can be directly detected myocardial ischemia, blood donor area and indirectly prompted severe coronary artery stenosis and the presence of lesions, mainly used in clinical routine screening and diagnosis of chronic stable coronary artery disease, the accuracy of $70 \%$ to $90 \%$ including exercise ECG, stress echocardiography and radionuclide myocardial perfusion imaging load. ECG exercise test include flat or treadmill exercise test. In patients with exercise load was observed ECG changes, detects whether induce myocardial ischemia, to diagnose coronary heart disease, the diagnostic accuracy of about $70 \%$. The advantage is simple, has been clinically routine screening for coronary heart disease [1]. However, ECG exercise test false positive and false negative rate, and early coronary artery stenosis less than about $70 \%$ of hard to find. Stress echocardiography is mainly based under load induced wall motion abnormality diagnosis of myocardial ischemia manifestations of coronary heart disease, the diagnostic accuracy of about $80 \%$, but not as good as exercise ECG is simple, not routine clinical application. Load radionuclide myocardial perfusion imaging is detected by the following load condition of myocardial perfusion defect that is reversible myocardial ischemia diagnosis of coronary heart disease, the diagnostic accuracy of $90 \%$, the clinical diagnosis of stable coronary artery disease the most accurate noninvasive method for the diagnosis value internationally recognized, US guidelines recommend clear load radionuclide myocardial perfusion imaging in particular as a coronary angiography interventional therapy "door man" [2], is widely used abroad, pharmacological stress and exercise stress myocardial perfusion imaging in the diagnosis of coronary artery disease sensitive and specificity was no significant difference. But its examination expensive and inconvenient to supply isotopes, radioactive, more limited domestic applications, most only routine use only in the provincial medical institutions.

Multislice spiral CT (MSCT). MSCT coronary angiography contrast agents directly through the display portion of coronary artery disease, the degree of stenosis, even nature, is the diagnosis of coronary artery stenosis and plaque latest non-invasive imaging method, the diameter $\geqslant 1.5 \mathrm{~mm}$ coronary segments, diagnostic coronary artery stenosis (> 50\%) with a sensitivity of $83 \%$ to $93 \%$ and specificity of $82 \%$ to $97 \%$, a positive predictive value of $71 \%$ to $83 \%$, and negative predictive value of $92 \%$ to $98 \%$. High negative predictive value may help clinicians rule out coronary artery stenosis, reduce the rate of misdiagnosis. By CT value, MSCT is also possible to determine the 
composition and nature of the organization in general coronary plaque, thereby enabling a preliminary assessment of the risk of plaque [3,4]. In addition, MSCT coronary angiography can show stenting or coronary bypass surgery luminal state, the position of the bracket shape, graft cases, for postoperative follow-up. MSCT Although it is currently a non-invasive and reliable technique for early diagnosis of coronary heart disease, but MSCT imaging will be affected heart rate, rhythm, coronary calcification, stents, pacemakers and other factors, there are still security problems of X-ray radiation, so checks should be strictly grasp the indications, should check the excesses, but cannot as a screening tool in clinical diagnosis of coronary heart disease.

Invasive coronary angiography and related technologies. Coronary angiography by selective coronary artery leaving the injection of contrast agent developed invasive techniques can directly display the coronary anatomy and lesion site of coronary artery stenosis and basic nature, is still the diagnosis of coronary heart disease and other coronary artery disease and most accurate way to check the gold standard; can confirm the diagnosis, treatment decisions; ll evaluate and predict long-term prognosis. Nevertheless, invasive coronary angiography, there may exist the risk of complications, the clinical indications should be strictly, not abuse. Further, the inner with percutaneous coronary intervention (percutaneous stransluminal coronary intervention, PCI) development and application of coronary intravascular ultrasound (intravascular ultrasound, IVUS) from endovascular and coronary artery disease show cross-sectional anatomy, morphology structure and properties, especially in recent years, concern about the vulnerable plaque, and plaque characterization of tissue resolution demanding virtual tissue imaging, optical coherence imaging (optical coherenttomography, OCT) and other new technologies to meet their requirements, because OCT better resolution (10 times IVUS, amounting to $10 \mu \mathrm{m}$ ), improves coronary plaque instability diagnostic capabilities and ability to risk stratification, and thus unstable plaque in the diagnosis and assessment better prospects [5].

\section{Early Diagnosis Strategy}

According to the pathophysiology of coronary heart disease, coronary heart disease diagnostic strategy has the following three points:

(1) Diagnosis of myocardial ischemia or infarction, rely mainly on the characteristics of the onset of symptoms and remission after the characteristic abnormal ECG changes and elevated cardiac enzymes or markers of myocardial injury; if they miss the opportunity to attack diagnosis you need through a variety of methods in the diagnosis of myocardial ischemia or myocardial infarction with or without the former cardiac load (exercise, drugs) test (ECG, echocardiography and radionuclide myocardial perfusion imaging), in cardiac stress-induced state and seized a reversible myocardial ischemia, the latter by electrocardiogram and myocardial enzymes evolution can be diagnosed.

(2) Identification of myocardial ischemia or myocardial infarction, early diagnosis is very important, mainly on the basis of onset of clinical features, the characteristic ECG changes and serum creatine kinase, abnormally elevated markers of injury, in which the clinical features of the most important, ECG changes are evidence, and serum creatine kinase and damage markers are final.

(3) A clear cause myocardial ischemia or infarction, coronary artery disease, which is the most direct evidence of anatomical diagnosis of coronary heart disease, mainly based on non-invasive coronary computed tomography scan (computerized tomography, CT) displayed directly, as well as invasive coronary accurate diagnosis (still the gold standard) angiography for chronic stable coronary artery disease, the key is "early" detect myocardial ischemia and severe coronary stenosis; for ACS, the key is "as soon as possible" diagnosis of myocardial ischemia or myocardial infarction, 
in order to aid and standard treatment. Especially the ACS, although many have prodromal symptoms, but very minor, easily overlooked and missed, early diagnosis of coronary heart disease is important and difficult.

\section{Conclusion}

In summary, patients with coronary artery disease early diagnosis, early detection and severity of the disease can be effectively suppressed development, and spectral sensitivity and specificity of the ECG as a good noninvasive means of early coronary artery disease can be effectively diagnosis, worthy of clinical use.

\section{Reference:}

[1] He Changping. Contrast Ecg And Coronary Angiography In The Diagnosis of Coronary Heart Disease [J]. Chinese Journal Of Gerontology, 2013,33 (19): 4830-4831.

[2] Liu Haiyan. Ecg St-T Segment Changes The Value Of Early Diagnosis of Coronary Heart Disease [J]. Foreign Medical Research, 2013 (32): 80-81.

[3] Cao Xiaoyu. Spectrum Ecg Coronary Heart Disease Early Diagnosis [J]. World Health Digest, 2011,07 (14): 69-70.

[4] Ma Mingxia. Seven Six Cases Crown Heart Disease Ecg Characteristics Analysis [J]. Health Road, 2014 (11): 401-402.

[5] Liugui Fen. Slice Spiral Ct In The Diagnosis of Coronary Heart Disease [J]. Medicine Heart Disease, 2014, (7): 831-832. 